\title{
When Utility Jumps: The Value of Having Cash in the Hand
}

\author{
Kurt W. Rotthoff ${ }^{*}$, Bentley Coffey ${ }^{2}$ \\ ${ }^{1}$ Seton Hall University, South Orange, NJ, USA \\ ${ }^{2}$ Duke University, Durham, NC, USA \\ Email: *rotthoff@gmail.com
}

How to cite this paper: Rotthoff, K.W. and Coffey, B. (2018) When Utility Jumps: The Value of Having Cash in the Hand. Theoretical Economics Letters, 8, 72-78. https://doi.org/10.4236/tel.2018.81004

Received: November 26, 2017

Accepted: January 22, 2018

Published: January 25, 2018

Copyright (c) 2018 by authors and Scientific Research Publishing Inc. This work is licensed under the Creative Commons Attribution International License (CC BY 4.0).

http://creativecommons.org/licenses/by/4.0/

\begin{abstract}
Different theoretical explanations have been developed for seemingly inconsistent actions that deal with varying levels of risk and time. We propose a simple model of utility that unifies these seemingly separate phenomena, while not departing too far from the standard models of utility maximization already in use. Our driving assumption is that preferences over riskier outcomes discontinuously depart from preferences under certainty; a jump from no risk to some risk is fundamentally different from a movement of some risk to more risk.
\end{abstract}

\section{Keywords}

Binary Jumps, Utility Theory, Risk

\section{Introduction}

Many different theoretical explanations of decision-making have been developed to characterize and judge experimental findings of bias as violations of standard utility theory. Explanations have come in the form of the certainty effect (also called the Allais Paradox), immediacy effect (also called present-bias, dynamic inconsistency, or diminishing impatience), utility of gambling, non-expected utility, risk aversion, and prospect theory. Work by Andreoni and Sprenger [1] suggests that models of preferences should be adjusted to accommodate a discrete taste for the absence of any sort of risk, which appears to be large enough to be empirically detectable. We attempt to unite these concepts via a simple adaptation of expected utility theory, which posits that agents behave as if maximizing their expected utility as described in the von Neumann and Morgenstern

*A special thanks to Robert Tollison, Bruce Yandle, Angela Dills, Michael Maloney, Pete Groothuis, Daniel Jones, and Hillary Morgan for helpful comments. Any mistakes made are our own. 
expected utility theorem (otherwise an agent's choices over uncertain lotteries might violate the independence axiom, implying that the individual would gladly succumb to predatory bets such as Dutch books).

Our value-added to literature is to propose that the presence of risk activates a discrete jump to a frame of mind that evaluates expected utility relative to some reference baseline. We implement this innovative idea in a model resembling a fusion of expected utility with quasi-hyperbolic utility, where the desired discrete jump is achieved via a discontinuity in the objective function at the boundary values of the probabilities. The inclusion of a discrete difference in riskless activities, relative to activities that include some positive level of risk, conveniently provides an efficient explanation of many experimental findings of bias.

We argue that all decisions begin with a binary choice: people either choose to take the action in question or not. To abstract away from the intellectual baggage that we carry from how economists have modelled risk, focus for a moment on the action to consume some good. Consider, for example, the decision to consume alcohol. The individual first decides whether to consume a taste of alcohol. Then, in a second stage, the individual decides how much more alcohol to consume on the margin. Economists might describe this binary decision of whether to consume alcohol is made on a coarse (i.e. discrete) margin; yet, it may be viewed differently by the decision maker from the fine (i.e. continuous) margins of (infinitesimally) tiny tweaks in the quantity consumed. Hence, the decision from none to some may be qualitatively different than the step from some to more. Now apply that same logic to a risk averse decision maker who is considering bearing some risk, the disutility of going from no risk to some risk can be distinctly different than going from some risk to more risk.

The classic example has an individual choosing between $\$ 100$ at time $t$ and $\$ 110$ at time $t+1$ chooses differently depending on the timing of these payments. When the decision is between $\$ 100$ today and $\$ 110$ in one month, people tend to choose $\$ 100$ today. However, if the decision is between $\$ 100$ in one year and $\$ 110$ in one year and one month, most people choose the latter [2]-[7]. While the gap in payments (one month) and the gap in pay (\$10) remain constant, the risk level does not. Payment today involves a riskless decision, whereas, all the other options involve some non-zero level of risk (although the experimenters hope that their design makes later payments appear riskless, the fact that the participant leaves without the money in hand means that they likely believe there is a non-zero probability of non-payment).

It is instructive to apply our logic to a practical example. If I hand you \$10,000 in cash, then say you can either a) keep it, or b) give it back (but I will give it back to you later with more money), which option do you take? It would depend on how much extra I give you back and your perceived risk of me taking it back. Could I offer you $\$ 1$ to take a small amount of risk? $\$ 10$ ? For most people, there is a minimum level of money that would have to be offered to take on the first level of risk (i.e. for any person to be willing for that money to leave their hand, there is some [non-small, non-linear] payment that would have to occur in order 
for them to take a positive level of risk). The initial movement from no risk to some risk is fundamentally different than the movement from some risk to more risk. The next section sets up the model and describes how these discrete utilities work. The last section concludes.

\section{Model}

We begin with a general specification of the decision maker's objective, as an (indirect) utility function $(V)$ that is increasing in the wealth $(W)$ owned in each of J states of nature $(S)$ :

$$
V\left(\left\{\operatorname{Pr}\left(S_{j}\right), W\left(S_{j}\right)\right\}_{j=1}^{J}\right)
$$

Then we propose the following functional form:

$$
V=U\left(W_{B}\right)+\beta^{1\left\{\max _{j} \operatorname{Pr}\left(S_{j}\right)<1\right\}}\left[\sum_{j=1}^{J} \operatorname{Pr}\left(S_{j}\right) U\left(W\left(S_{j}\right)\right)-U\left(W_{B}\right)\right]
$$

where $U$ is a state-dependent utility function under certainty, $W_{B}$ denotes the amount of wealth which serves as a baseline for the decision-maker, and $\beta \in[0,1]$ represents the penalty to the decision-maker from the presence of risk. ${ }^{1}$ The baseline reference is the threshold at which the agent is indifferent between a risky gamble and a certain outcome with the same expected value (so that the agent is risk averse above the baseline reference and risk seeking below it); operationally, $\mathrm{W}_{\mathrm{B}}$ is just a preference parameter.

We immediately note three desirable properties about this specification.

Observation 1. Our specification nests von Neumann-Morgenstern Expected Utility as a special case when $\beta=1$ :

$$
V=\sum_{j=1}^{J} \operatorname{Pr}\left(S_{j}\right) U\left(W\left(S_{j}\right)\right) \quad \text { if } \beta=1
$$

Observation 2. When the uncertainty distribution is degenerate, our specification neatly collapses to utility under certainty:

$$
V\left(\left\{\operatorname{Pr}\left(S_{A}\right)=1, W\left(S_{A}\right) ; \cdots\right\}\right)=U\left(W\left(S_{A}\right)\right)
$$

Observation 3. When the present is certain and the future is inherently uncertain, then the time separable version of our preferences conforms to the model of preferences that exhibit quasi-hyperbolic discounting:

$$
V_{0}=U_{0}+\sum_{t=1}^{T} \delta^{t}\left[\beta^{1\left\{\max _{j} \operatorname{PrPr}\left(S_{j t}\right)<1\right\}}\left[\sum_{j=1}^{J} \operatorname{Pr}\left(S_{j t}\right) U\left(W\left(S_{j t}\right)\right)-U\left(W_{B}\right)\right]\right]
$$

Hence, any phenomenon explained with quasi-hyperbolic discounting also explains our preferences that anchor expected utility to a reference baseline. The hyperbolic discounting parameter appears due to our model of a discrete jump

${ }^{1}$ Where the term "risk" is used to mean that there is uncertainty (i.e. a non-degenerate probability distribution) over outcomes that the decision maker strictly orders (this excludes the uninteresting case of uncertainty over outcomes for which the decision maker is indifferent). 
in utility when moving from certainty (at the present) to uncertainty (of the future). In our model, the additional discounting of the future can give an intimately tied intuitive interpretation to disutility due to the mere presence of uncertainty in the future.

Observation 4. By design, our specification produces a discontinuity between certainty and uncertainty at any arbitrary wealth level, $W\left(S_{A}\right)$, apart from the baseline, when $\beta \in(0,1)$ :

$$
\begin{gathered}
V\left(\left\{\operatorname{Pr}\left(S_{A}\right)=1, W\left(S_{A}\right) ; \cdots\right\}\right) \neq \lim _{\operatorname{Pr} \operatorname{Pr}\left(S_{A}\right) \rightarrow 1} V\left(\left\{\operatorname{Pr}\left(S_{A}\right), W\left(S_{A}\right) ; \cdots\right\}\right) \\
U\left(W\left(S_{A}\right)\right) \neq \beta U\left(W\left(S_{A}\right)\right)+(1-\beta) U\left(W_{B}\right)
\end{gathered}
$$

Notice that, even when there is some fleetingly small risk, utility is a convex combination of the utility of a von Neumann Morgenstern Expected Utility maximizer and some baseline frame of reference to which this decision maker is tethered. The strength of that tether is determined by the magnitude of $\beta$ : the closer the parameter for a decision maker is to 1 , the closer the decision-maker is to being a pure von Neumann Morgenstern Expected Utility maximizer. The closer the parameter for a decision maker is to 0 , the closer the decision-maker is to appearing somewhat irrational relative to the von Neumann Morgenstern model. Our prior is that likely values for $\beta$ will tend to be rather close to (albeit just less than) 1 . To clearly illustrate the mechanics of this model of preferences, Figure 1 depicts the mechanics for an exaggerated value of the $\beta$ parameter $(\beta \approx$ $0.5)$.

Figure 1 plots indirect utility in units of utils on the vertical axis versus dollar-denominated wealth on the horizontal axis. The green curve is a standard utility function under certainty. The blue horizontal line is the reference level of utility, which crosses the standard utility function at the point of reference (labeled $W_{B}$ ). Above this point, anchoring to the reference point makes the decision maker relatively more risk averse but less risk averse below this reference point. The blue curve is just the weighted average of the green curve and the horizontal blue line. The jump from uncertainty to certainty induces a discrete gain in utility above the reference point but a discrete drop in utility below the reference point. The standard graphical exercises can be conducted with any state dependent utility function (e.g. between an outcome yielding $W_{L}$ versus $W_{H}$ ), but one must then anchor it to the reference level of utility.

In Figure 2, we analyze how an agent with these preferences would change their valuations of risky outcomes due to a change in the probability of increasing wealth from $W_{L}$ to $W_{H}$. When the amounts of wealth in question are above the baseline reference (i.e. $W_{H}>W_{L}>W_{B}$ ), then the presence of any uncertainty in the amount of wealth decreases the individual's valuation. When the amounts of wealth in question are below the baseline reference (i.e. $W_{B}>W_{H}>W_{L}$ ), then the presence of some uncertainty in the amount of wealth actually increases the individual's valuation. When the amounts straddle the baseline (i.e. $W_{B}>W_{H}>$ $W_{L}$ ), then the presence of risk contracts the valuations toward that baseline. 


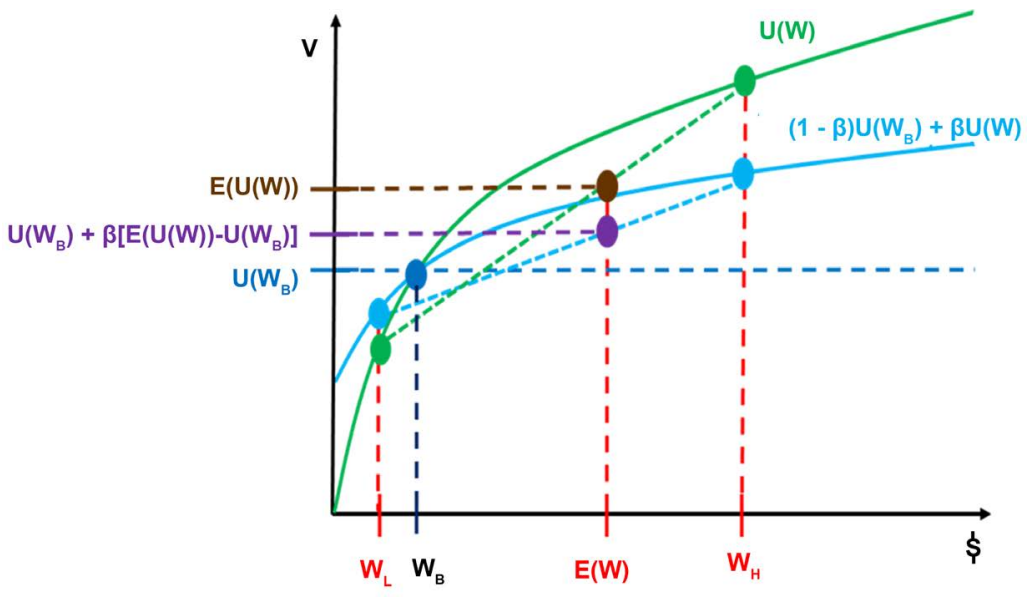

Figure 1. Depicting our proposed augmentation of the standard expected utility model with a discrete distaste for extensive risk.


Figure 2. How our proposed discrete distaste for extensive risk relates to the reference point.

Note that this middle case appears to resemble a stylized form of the weighting function proposed in the prospect theory of Kahneman and Tversky [4], which famously used a sigmoidal shape. Thus, in some sense, our model could be seen as proposing a clever weighting function for prospect theory that nicely yields quasi-hyperbolic preferences.

Figure 3 depicts how the preferences would appear in a canonical figure from finance: indifference curves between portfolios of various combinations of risk and return as the mean return versus the variance of returns. The indifference curves resemble what we draw from the standard von Neumann Morgenstern expected utility decision-making model; the difference appears in the discontinuities in the intercept. For amounts in excess of the baseline reference, the presence of any risk clearly generates a discrete drop in utility. For amounts beneath the baseline reference, the presence of some risk can enhance utility. This feature can explain how gambling small amounts of money, so long as the amounts in question fall beneath the baseline reference, can actually enhance utility. Indeed, we intuitively conceptualized the reference level as the level beneath which there exists some risky gamble that would be preferred to a certain 


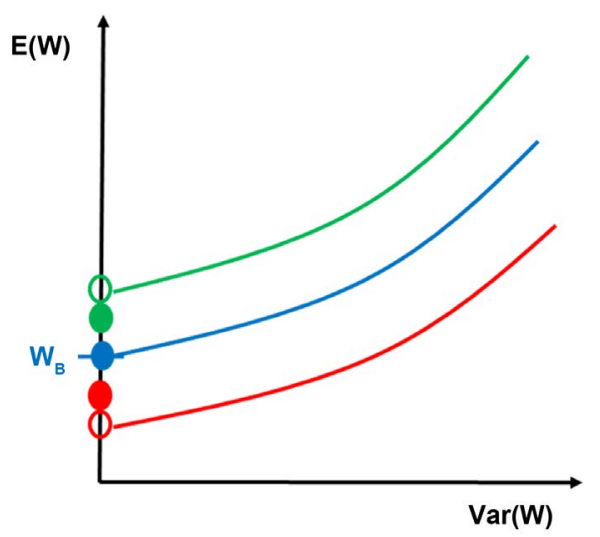

Figure 3. How our proposed discrete distaste for extensive risk relates to the reference point.

outcome with the same expected value. It is certainly conceivable that this reference baseline may change over time, inducing a source of time inconsistency for a longer run scope than the simpler form captured by hyperbolic discounting (i.e. the present versus the future), for reasons that we do not explore here.

\section{Conclusion}

Engaging in risky activities is inevitable. Virtually all decisions entail some level of risk; the ability to eliminate all risk is relatively rare and hence very valuable. We have constructed a parsimonious model that captures the discrete jump in utility from selecting a risk-free option. With this discrete jump achieved via a discontinuity in the objective function at the boundary values of the probabilities, our model includes familiar features of both expected utility and quasi-hyperbolic utility (which are special cases). Our model provides a unifying and consistent explanation for a variety of anomalous behavior associated with behavioral biases: certainty effect, Allais Paradox, immediacy effect, present-bias, dynamic inconsistency, diminishing impatience, the utility of gambling, non-expected utility, and prospect theory. We encourage future research to continue to refine the use of this discrete utility function, consider the additional applications, and pursue further estimations of its parameters. Although there are limitations on this application, as there is for any application of utility theory, the ability to unify these different theories opens the door to many avenues of future research.

\section{References}

[1] Andreoni, J. and Sprenger, C. (2012) Risk Preferences Are Not Time Preferences. American Economic Review, 102, 3357-3376. https://doi.org/10.1257/aer.102.7.3357

[2] Friedman, M. and Savage, L.J. (1948) The Utility Analysis of Choices Involving Risk. The Journal of Political Economy, 56, 279-304.

[3] Phelps, E. and Pollak, R. (1968) On Second-Best National Saving and Game-Equilibrium Growth. Review of Economic Studies, 35, 185-199. https://doi.org/10.2307/2296547

[4] Kahneman, D. and Tversky, A. (1979) Prospect Theory: An Analysis of Decision 
under Risk. Econometrica, 47, 263-292.

[5] Laibson, D. (1997) Golden Eggs and Hyperbolic Discounting. Quarterly Journal of Economics, 112, 443-477.

[6] O’Donoghue, T. and Rabin, M. (1999) Doing It Now or Later. The American Economic Review, 89, 1030-1124.

[7] Benhabib, J., Bisin, A. and Schotter, A. (2010) Present-Bias, Quasi-Hyperbolic Discounting, and Fixed Costs. Games and Economic Behavior, 69, 205-223.

https://doi.org/10.1016/j.geb.2009.11.003 